\title{
FORMULATION DESIGN AND CHARACTERIZATION OF ROPINIROLE HYDROCHORIDE MICROSPHERE FOR INTRANASAL DELIVERY
}

\author{
SHUBHRAJIT MANTRY ${ }^{1 *}$, ANNA BALAJI ${ }^{2}$ \\ ${ }^{1}$ Department of Pharmaceutics, Research Scholar, Institute of Pharmaceutical Sciences and Research Center, Bhagwant University, \\ Ajmer, Rajasthan, India. ${ }^{2}$ Department of Pharmaceutics, Pathfinder Institute of Pharmacy Education \& Research. Beside Mamnoor \\ Camp, Khammam Road, Warangal, Telangana, India. Email: manu28pharmacy@gmail.com
}

Received: 15 March 2017, Revised and Accepted: 13 April 2017

\section{ABSTRACT}

Objective: The objective of this research work to design nasal microspheres of ropinirole hydrochloride (HCL) using different mucoadhesive polymers by adopting the suitable technique. To study the influence of formulation and process variables on microsphere formation and release characteristics. To perform the physicochemical characterization of the prepared microspheres. To carry out in vitro drug release studies and to explore the release behavior using various kinetic models.

Methods: Experiments were performed with ropinirole HCL as a drug, chitosan, guar gum, carbopol 974P as a polymer. Span 80 and Tween 80 used light liquid paraffin, concentrated hydrochloric acid as solvent.

Result: The in vitro drug release studies were conducted for all the formulations, that is, F1-F21 in $250 \mathrm{ml}$ phosphate buffer pH 6.6 for 12 hrs. Among them, F15 showed $82.7 \pm 0.23 \%$ drug release and F21 showed $81.2 \%$ in $12 \mathrm{hrs}$ in a sustained manner.

Conclusion: Microspheres were formulated by emulsion solvent evaporation technique using different polymers. Apart from preventing nasal irritation, the microsphere possesses two major advantages over plain solutions, one is a high solubilization capacity for ropinirole HCL that exceeds their aqueous solubility and thus allows a reduction in the application volume. The results of this work indicate that intranasal microsphere of ropinirole may be beneficial for the treatment of Parkinson's disease.

Keywords: Ropinirole hydrochloride, Parkinson's disease, Microspheres.

(c) 2017 The Authors. Published by Innovare Academic Sciences Pvt Ltd. This is an open access article under the CC BY license (http://creativecommons. org/licenses/by/4. 0/) DOI: http://dx.doi.org/10.22159/ajpcr.2017.v10i7.18475

\section{INTRODUCTION}

Microspheres are solid spherical particles ranging in size from 1 to $1000 \mu \mathrm{m}$. They are spherical free flowing particles comprising proteins or synthetic polymers. The microspheres are free flowing powders including proteins or synthetic polymers, which are biodegradable in nature. There are two types of microspheres; microcapsules and micromatrices, which are described as microcapsules are those in which entrapped substance is distinctly surrounded by distinct capsule wall and micromatrices in which entrapped substance is dispersing throughout the microspheres matrix. Solid biodegradable microspheres incorporating a drug dispersed or dissolved through particle matrix have the potential for the controlled release of the drug. They are made up of polymeric, waxy, or other protective materials, that is, biodegradable synthetic polymers and modified natural products [1].

Delivery of drug into the brain for central nervous system (CNS) disease require treatment, however, such route of delivery is very problematic. Therapeutic effect of the drug can be described by achieving the desired concentration of drug in blood or tissue for a prolonged period. Hence, it is a reliable means to deliver a drug to a target site with specificity and in a controlled manner. Microsphere used as a not controlled release but for targeted therapy also so it offers certain advantages over the conventional release dosage form for those drugs having a first pass metabolism. All these drawbacks of conventional delivery system necessitate the development of controlled drug delivery system. Delivery of drug to CNS is problematic for drugs having a hydrophilic in nature and having a high molecular weight because of impervious nature of Blood-Brain Barriers (BBB) [2].
Parkinsonism disease is a leading cause of neurological disability and is the second most common progressive neurodegenerative disorder. The effect of the Parkinsonism disease reaches $1-2 \%$ in people over the age of 50. It has no gender preference and has a worldwide distribution. The symptoms of Parkinsonism disease are largely related to progressive loss of dopamine in the basal ganglia [3].

While enormous progress has been made regarding our understanding of the pathogenic mechanisms of neurological diseases, there are only a small number of effective drugs for treating this illness. A key obstacle for developing an effective treatment for neurological diseases is the blockage of drug entrance into the CNS by the BBB. $<2 \%$ of all small molecule-drugs and virtually no large molecule drugs can cross the BBB. Therefore, it is of critical significance to search drug delivery strategies that can effectively deliver the drugs to CNS [4].

Recent developments in nasal drug delivery have suggested intranasal administration as a safe and acceptable route for brain targeting, especially for drugs with biological effects on the CNS and limited blood-brain permeability [5].

The nose to brain delivery would be beneficial in therapeutic situations where a rapid and/or specific targeting of drugs to the brain is required. Conditions such as Parkinson's disease, Alzheimer's disease or pain would be benefited from the development of nasal delivery systems, which will increase the fraction of drug that reaches the CNS after nasal delivery. The olfactory region located at the upper remote parts of the nasal passages offers the potential for certain compounds to circumvent the BBB and enter into the brain [6]. 
Intranasal delivery also offers the advantage of simple administration, cost effectiveness and convenient. This novel delivery method allows drugs, therapeutic proteins, polynucleotides, and viral vectors that do not normally cross the BBB to be delivered to the CNS. In addition, intranasal targeting of drugs to the CNS avoids first pass elimination by the liver allowing a lower therapeutic drug dose and fewer systemic side effects. Delivery from the nose to the CNS occurs within minutes along both the olfactory and trigeminal nerves. Delivery occurs by an extracellular route and does not require that the drugs bind to any receptor or undergo axonal transport [7-12].

Ropinirole, a recent introduction in the clinical treatment of Parkinson's disease, suffers with the problems of low oral bioavailability and frequent dosing [13].

It is an orally administered non-ergoline dopamine agonist, and chemically it is hydrochloride (HCL) salt of 4-[2-(dipropyl amino) ethyl]-1, 3-dihydro-2H-indol-2-one with a molecular weight of 296.84. It is having short half-life (4-6 hrs) and low bioavailability (35\%) due to extensive first pass metabolism [14].

The usual dose is $3-9 \mathrm{mg}$ daily and has to be taken in three divided doses due to a short half-life of the drug [15].

Microspheres are solid spherical particles ranging from 1 to 1000 $\mu \mathrm{m}$. Bioadhesive microspheres give more residence time to facilitate absorption through nasal mucosa against nasal mucociliary clearance [1].

The treatment of all neurodegenerative diseases is a big challenge because of the numerous protective barriers surrounding the CNS. The targeting of drug to the CNS, for the therapeutic advancement of neurodegenerative disorders such as Alzheimer's and Parkinson's disease can be done by administering the drug formulation such as nanoparticle, liposome, microemulsion, and microsphere, which can cross the BBB or by delivering the drug formulation through intranasal route which can bypass the BBB [16].

Nasal delivery of drugs targeting the CNS is currently an area of great interest. In addition to "nose to brain delivery" intranasal drugs can enter through a "nose to systemic circulation to brain" pathway. In this case, it is necessary for the drug to readily permeate the BBB from the circulation. For this to be achieved the drug must exhibit satisfactory passive or active transport across the light junction barriers of the BBB [17].

\section{MATERIALS AND METHODS}

\section{Materials}

Experiments were performed with ropinirole HCL as a drug obtained from Hetero pharma, Hyderabad, India. Chitosan, guar gum, carbopol 974P as a polymer purchased from Finar chemicals limited, Ahmedabad and Burgoyne Burbridges and Co., Mumbai, India. Span 80 and Tween 80 used as solvent purchased from Finar Chemicals Limited, Ahmedabad, India. Light liquid paraffin, concentrated hydrochloric acid obtained from Molychem, Mumbai, India.

\section{Methods}

Formulation of intranasal hydrogel microbeads of ropinirole HCL Trial and error method

(Preliminary experiments) previously many trials were run for the preparation of microbeads of ropinirole HCL by emulsion solvent evaporation technique using different polymers. Trials were made by changing the temperature, stirring speeds, the concentration of the polymer, Tween-80, Span-80. After so many trials, it was concluded that temperature play a very critical role in the formation of microbeads, it is a continuous process of stirring, with the combination of hydrophilic and lipophilic surfactants. Every step in the process was optimized by performing experiments through trial and error method.

\section{Preparation of ropinirole HCL microspheres}

Ropinirole HCL microspheress are prepared by emulsion solvent evaporation technique using three polymers, that is, chitosan, carbopol 974P and guar gum individually and in combinations. The following are the steps for the preparation of microbeads. Polymer (chitosan, carbopol 974P and guar gum) was allowed to hydrate in $40 \mathrm{ml}$ of water for some time to achieve a viscous solution. Weighed quantity of ropinirole HCL was dispersed in $10 \mathrm{ml}$ of water and this was added to the polymer dispersion. To the above drug-gum dispersion $0.1 \%$ Tween 80 was added under constant stirring. Separately $200 \mathrm{ml}$ of light liquid paraffin was measured in a glass beaker and to this $0.1 \%$ Span 80 was added to it. The oil phase was placed on a hot plate fitted with a remi stirrer, and aqueous phase/polymer dispersion was added to the oil phase in a thin stream over 2-3 minutes. This emulsion was stirred at $2000 \mathrm{rpm}$ and heated to $80^{\circ} \mathrm{C}$ for $3-4 \mathrm{hrs}$. The aqueous phase evaporates leaving the microspheres dispersed in the oil phase. Microspheres were harvested by decantation from the oil phase and washed 3-4 times with $100 \mathrm{ml}$ aliquots of $\mathrm{n}$-Hexane to make the Microbeads free from oil. Then, these are allowed to dry at $40^{\circ} \mathrm{C}$ for $1 \mathrm{hr}$ in a hot air oven. All the microbeads were stored in tight containers till taken for further evaluation (Table 1).

\section{RESULTS AND DISCUSSION}

A total of 21 formulations of ropinirole HCL microspheres with various polymers were formulated individually and in combination by an emulsion solvent evaporation technique. The formulations were subjected to evaluation parameters such as particle size, surface morphology, drug entrapment efficiency (DEE), Swelling studies, in-vitro mucoadhesive studies, and in-vitro drug release studies.

\section{Standard calibration curve of ropinirole HCL}

Preparation of standard stock solution:

$100 \mathrm{mg}$ of ropinirole HCL was accurately weighed and dissolve into $100 \mathrm{ml}$ volumetric flask containing $\mathrm{pH} 6.6$ buffer solution to get a concentration of $(1000 \mu \mathrm{g} / \mathrm{ml})$, that is, stock solution-I. from this $1 \mathrm{ml}$ was withdrawn and diluted to $100 \mathrm{ml}$ with $\mathrm{pH} 6.6$ phosphate buffer, to get a concentration of $(10 \mu \mathrm{g} / \mathrm{ml})$, that is, stock solution-II.

\section{Calibration curve of pH 6.6 phosphate buffer solution}

From the stock solution-II, 2,4,6,8, and $10 \mathrm{ml}$ sample were withdrawn and volume was made up to the mark with $\mathrm{pH} 6.6$ phosphate buffer. This solution gives $2,4,6,8$, and $10 \mu \mathrm{g} / \mathrm{ml}$ concentration of ropinirole HCL. The absorbance of these solutions measured in UV at $250 \mathrm{~nm}$ using pH 6.6 phosphate buffer as blank. Standard calibration curve of ropinirole HCL in $6.6 \mathrm{pH}$ phosphate buffer solution at $\lambda$ max $250 \mathrm{~nm}$ (Table 2 and Fig. 1).

\section{Characterization of ropinirole HCL microspheres}

\section{Micromeritic properties of microspheres}

All the formulations F1-F21 are evaluated for bulk density, tapped density, Carr's compressibility index, and Hausner's ratio. Compressibility, that is, Carr's index, was found to be between $5.17 \%$ and $22.8 \%$ and Hausner's ratio was found to be between 1.05 and 1.29 , all the parameters indicating good flow property (Table 3).

\section{Percentage yield}

Percentage yield of different formulations, F1-F21, were calculated and the yield was found to be in the range of $86-98.9 \%$, respectively. This higher percentage yield indicates that this emulsion solvent evaporation method was very useful for adoption in the formulation of ropinirole HCL microspheres.

\section{Particle size analysis}

Particle size distribution of ropinirole HCL microspheres was determined by optical microscope fitted with an ocular micrometer and stage micrometer. All the formulations of micro beads F1-F21 show uniform size distribution. The average particle size of ropinirole HCL 
Table 1: Formulation of ropinirole HCL microspheres

\begin{tabular}{|c|c|c|c|c|c|c|c|}
\hline Formulation code & Drug (mg) & Chitosan \%(W/V) & $\begin{array}{l}\text { Carbopol 974P } \\
\%(W / V)\end{array}$ & $\begin{array}{l}\text { Guar gum } \\
\%(W / V)\end{array}$ & Tween $80 \%$ & Span $\mathbf{8 0} \%$ & $\begin{array}{l}\text { Liquid } \\
\text { paraffin }(\mathrm{ml})\end{array}$ \\
\hline F1 & 50 & 1 & - & - & 0.1 & 0.1 & 200 \\
\hline F2 & 50 & 1.5 & - & - & 0.1 & 0.1 & 200 \\
\hline F3 & 50 & 2.5 & - & - & 0.1 & 0.1 & 200 \\
\hline F4 & 50 & 3.5 & - & - & 0.1 & 0.1 & 200 \\
\hline F5 & 50 & 5 & - & - & 0.1 & 0.1 & 200 \\
\hline F6 & 50 & - & 1 & - & 0.1 & 0.1 & 200 \\
\hline F8 & 50 & - & 2 & - & 0.1 & 0.1 & 200 \\
\hline F9 & 50 & - & 2.5 & - & 0.1 & 0.1 & 200 \\
\hline F10 & 50 & - & 3.5 & - & 0.1 & 0.1 & 200 \\
\hline F11 & 50 & - & - & 1 & 0.1 & 0.1 & 200 \\
\hline F12 & 50 & - & - & 1.5 & 0.1 & 0.1 & 200 \\
\hline F13 & 50 & - & - & 2 & 0.1 & 0.1 & 200 \\
\hline F14 & 50 & - & - & 2.5 & 0.1 & 0.1 & 200 \\
\hline F15 & 50 & - & - & 3.5 & 0.1 & 0.1 & 200 \\
\hline F17 & 50 & 2.5 & - & 2.5 & 0.1 & 0.1 & 200 \\
\hline F18 & 50 & 1.75 & 1.75 & - & 0.1 & 0.1 & 200 \\
\hline F19 & 50 & 2.5 & 2.5 & - & 0.1 & 0.1 & 200 \\
\hline F20 & 50 & - & 1.75 & 1.75 & 0.1 & 0.1 & 200 \\
\hline F21 & 50 & - & 2.5 & 2.5 & 0.1 & 0.1 & 200 \\
\hline
\end{tabular}

HCL: Hydrochloride

\section{Table 2: Standard plot of ropinirole HCL in 6.6 phosphate buffer}

\begin{tabular}{ll}
\hline Concentration $(\mathrm{mcg} / \mathrm{ml})$ & Absorbance \\
\hline 0 & 0 \\
2 & 0.071 \\
4 & 0.139 \\
6 & 0.216 \\
8 & 0.284 \\
10 & 0.361 \\
\hline
\end{tabular}

HCL: Hydrochloride

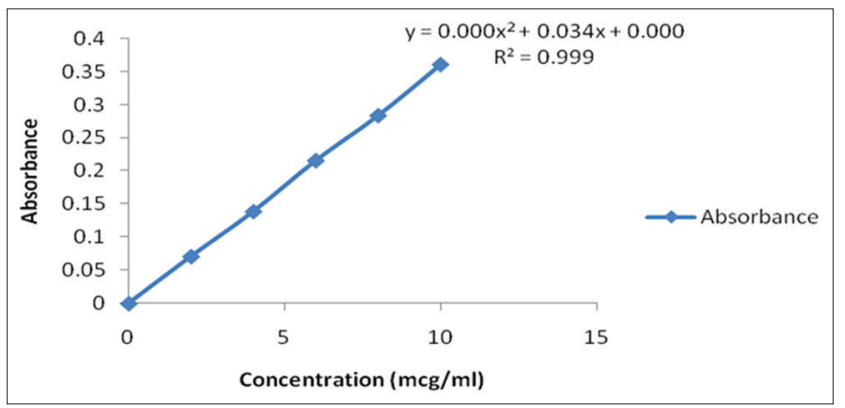

Fig. 1: Standard graph

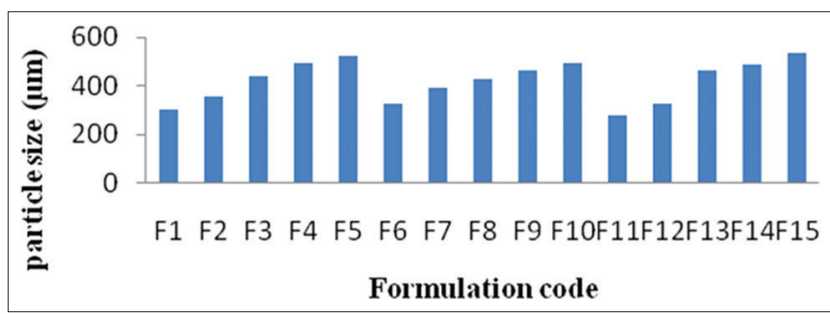

Fig. 2: Comparison of results of particle size analysis between F1 and F15 formulations

microspheres was found to be in the range of $280 \pm 3.15-535 \pm 2.28 \mu \mathrm{m}$. As the polymer concentration was increased, the size of the microspheres was also found to be increased.

\section{Drug entrapment efficiency (DEE)}

The entrapment process was found to be good and in the range of $61.1 \pm 2.5-94.4 \pm 1.38 \%$ for the formulations F1-F15 and $83.4 \pm 2.43$ $95.3 \pm 2.81 \%$ for the formulations F16-F21. The percentage of entrapment was higher, $94.4 \pm 1.38 \%$ for F5 ( $5 \%$ chitosan), $92.3 \pm 1.91 \%$ for F10 (3.5\% carbopol 974P) and $93.7 \pm 1.28 \%$ for F15 (3.5\% guar gum) formulation. This improved entrapment efficiency is simply due to the greater proportion of polymers with respect to the amount of drug.

Loose surface crystallography (LSC)

LSC studies were conducted for all the drug-loaded formulations F1F15 and F16-F21. Surface-associated drug content of microspheres decreased with increase in the concentration of the polymer. As the polymer concentration increased from F1-F5, F6-F10 and F11-F15 it showed increased entrapment efficiencies and hence decreased surface drug contents. However in formulations with low polymer concentration the surface associated drug content was more in F1 (30.22\%), F6 (32.13\%) and F11 (25.01\%) formulations due to the lower entrapment efficiency. The surface associated drug content for combination formulations, F16-F21 ranged between $10 \%$ and $18.8 \%$ (Table 4 and Figs. 2-5).

\section{Swelling index (SI)}

Swelling property was mostly affected by the concentration of polymer. As the concentration of polymers, that is, chitosan, carbopol 974P and guar gum increases the swelling capacity increased. The SI for all the formulations, that is, F1-F21 was determined in $\mathrm{pH} 6.6$ phosphate buffer. The SI increased from F1 (175.3 $\pm 8.07 \%)$ to F5 (55.2 $\pm 12.6 \%)$, F6 $(169.2 \pm 8.3 \%)$ to $\mathrm{F} 10(311.4 \pm 11.25 \%), \mathrm{F} 11 \quad(159.8 \pm 9.13 \%)$ to $\mathrm{F} 15(330.5 \pm 11.1 \%)$ in $\mathrm{pH} 6.6$ buffer at the end of 2 hrs. The increase in the SI is due to the increase in the polymer concentration. The swelling capacity in $\mathrm{pH} 6.6$ phosphate buffer is more which indicated the greater swelling capacity in alkaline medium. The SI of F16-F21 formulations ranged from $270 \pm 8.34 \%$ to $350 \pm 9.6 \%$ in the $\mathrm{pH} 6.6$ buffer at the end of $2 \mathrm{hrs}$. This is due to the higher concentration of combination polymers due to the combinations of polymers (Table 5).

\section{In vitro drug release studies}

The in vitro drug release studies were conducted for all the formulations, that is, F1-F21 in $250 \mathrm{ml}$ phosphate buffer $\mathrm{pH} 6.6$ for 
Table 3: Results of the micrometric properties of formulations F1-F21

\begin{tabular}{|c|c|c|c|c|}
\hline Formulation code & Bulk density (g/cc3) & Tapped density (g/cc3) & Compressibility index (\%) & Hausners ratio \\
\hline $\mathrm{F} 1$ & $0.26 \pm 0.012$ & $0.31 \pm 0.023$ & $16.1 \pm 1.33$ & $1.19 \pm 0.03$ \\
\hline F2 & $0.28 \pm 0.083$ & $0.38 \pm 0.032$ & $21.3 \pm 0.63$ & $1.25 \pm 0.02$ \\
\hline F3 & $0.41 \pm 0.033$ & $0.47 \pm 0.010$ & $12.76 \pm 1.34$ & $1.14 \pm 0.13$ \\
\hline $\mathrm{F} 4$ & $0.29 \pm 0.032$ & $0.35 \pm 0.009$ & $17.14 \pm 1.38$ & $1.2 \pm 0.03$ \\
\hline F5 & $0.5 \pm 0.062$ & $0.55 \pm 0.033$ & $9.09 \pm 1.22$ & $1.1 \pm 0.02$ \\
\hline F6 & $0.33 \pm 0.092$ & $0.41 \pm 0.023$ & $19.51 \pm 1.68$ & $1.24 \pm 0.03$ \\
\hline F7 & $0.28 \pm 0.083$ & $0.38 \pm 0.032$ & $21.3 \pm 0.63$ & $1.25 \pm 0.02$ \\
\hline F9 & $0.27 \pm 0.040$ & $0.35 \pm 0.009$ & $22.8 \pm 1.77$ & $1.29 \pm 0.10$ \\
\hline F10 & $0.62 \pm 0.033$ & $0.71 \pm 0.034$ & $12.6 \pm 0.72$ & $1.14 \pm 0.04$ \\
\hline F11 & $0.26 \pm 0.012$ & $0.31 \pm 0.023$ & $16.1 \pm 1.33$ & $1.19 \pm 0.03$ \\
\hline F12 & $0.41 \pm 0.033$ & $0.47 \pm 0.010$ & $12.76 \pm 1.34$ & $1.14 \pm 0.13$ \\
\hline F13 & $0.45 \pm 0.062$ & $0.5 \pm 0.052$ & $10 \pm 0.56$ & $1.11 \pm 0.12$ \\
\hline F14 & $0.5 \pm 0.062$ & $0.55 \pm 0.033$ & $9.09 \pm 1.22$ & $1.1 \pm 0.02$ \\
\hline F15 & $0.29 \pm 0.042$ & $0.35 \pm 0.023$ & $17.14 \pm 1.63$ & $1.2 \pm 0.18$ \\
\hline F16 & $0.45 \pm 0.023$ & $0.5 \pm 0.023$ & $10 \pm 1.33$ & $1.11 \pm 0.02$ \\
\hline F18 & $0.62 \pm 0.062$ & $0.66 \pm 0.013$ & $6.06 \pm 1.99$ & $1.06 \pm 0.13$ \\
\hline F19 & $0.55 \pm 0.033$ & $0.58 \pm 0.013$ & $5.17 \pm 0.77$ & $1.05 \pm 0.16$ \\
\hline F20 & $0.17 \pm 0.064$ & $0.29 \pm 0.024$ & $21.3 \pm 1.45$ & $1.25 \pm 0.03$ \\
\hline F21 & $0.28 \pm 0.083$ & $0.38 \pm 0.032$ & $21.3 \pm 0.63$ & $1.25 \pm 0.02$ \\
\hline
\end{tabular}

Each sample was analyzed in triplicate $(\mathrm{n}=3 \pm \mathrm{SD})$, SD: Standard deviation

Table 4: Data of evaluation parameters of MS microbeads formulations F1-F21

\begin{tabular}{|c|c|c|c|c|}
\hline Formulation code & Yield (\%) & Particle size $(\mu \mathrm{m})$ & Drug entrapment efficiency (\%) & LSC (\%) \\
\hline F1 & 88.3 & $300 \pm 3.12$ & $65 \pm 1.03$ & $30.22 \pm 0.16$ \\
\hline F2 & 91.8 & $355 \pm 3.68$ & $77 \pm 2.22$ & $22.14 \pm 0.15$ \\
\hline F3 & 98.1 & $440 \pm 2.96$ & $84 \pm 2.4$ & $19.32 \pm 0.18$ \\
\hline F4 & 98.3 & $495 \pm 3.76$ & $87.5 \pm 3.32$ & $15.12 \pm 0.11$ \\
\hline F5 & 95.8 & $525 \pm 2.82$ & $94.4 \pm 1.38$ & $10.1 \pm 0.17$ \\
\hline F6 & 89 & $325 \pm 4.58$ & $61.1 \pm 2.5$ & $32.13 \pm 0.22$ \\
\hline F7 & 95.4 & $390 \pm 4.23$ & $77.6 \pm 2.17$ & $23.13 \pm 0.15$ \\
\hline F9 & 98.3 & $466 \pm 3.66$ & $89.2 \pm 2.35$ & $12.2 \pm 0.18$ \\
\hline F10 & 96.6 & $495 \pm 2.76$ & $92.3 \pm 1.91$ & $10.3 \pm 0.16$ \\
\hline F11 & 94.2 & $280 \pm 3.15$ & $75.7 \pm 1.33$ & $25.01 \pm 0.17$ \\
\hline F12 & 96.5 & $324 \pm 2.83$ & $81.2 \pm 2.42$ & $19.21 \pm 0.11$ \\
\hline F13 & 93.1 & $465 \pm 4.28$ & $88.9 \pm 2.01$ & $15.24 \pm 0.18$ \\
\hline F14 & 96.5 & $488 \pm 3.68$ & $91.2 \pm 3.12$ & $12 \pm 0.23$ \\
\hline F15 & 96 & $535 \pm 2.28$ & $93.7 \pm 1.28$ & $10.8 \pm 0.10$ \\
\hline F16 & 98.9 & $390 \pm 2.82$ & $84.1 \pm 1.29$ & $18.3 \pm 0.14$ \\
\hline F18 & 89.3 & $415 \pm 3.33$ & $83.4 \pm 2.43$ & $18.8 \pm 0.14$ \\
\hline F19 & 94 & $470 \pm 2.82$ & $95.1 \pm 3.31$ & $9.48 \pm 0.19$ \\
\hline F20 & 95.2 & $410 \pm 2.35$ & $85.4 \pm 1.35$ & $17.8 \pm 0.18$ \\
\hline F21 & 86 & $512 \pm 3.66$ & $95.3 \pm 2.81$ & $9.22 \pm 0.11$ \\
\hline
\end{tabular}

Each sample was analyzed in triplicate $(n=3 \pm S D)$, SD: Standard deviation

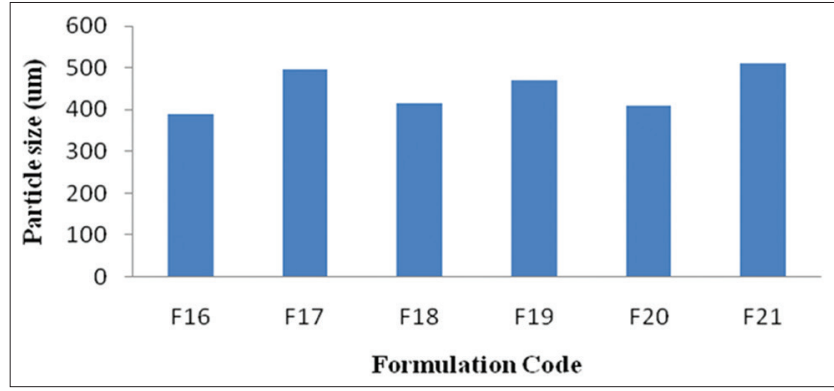

Fig. 3: Comparison of results of particle size analysis between F16 and F21 formulations

12 hrs. The percentage of drug release for formulations F1-F5 were found to be in the range of $86.4 \pm 1.22-98.9 \pm 0.54 \%$ the maximum drug release was found to be $98.9 \pm 0.54 \%$ in formulation F1 in 12 hrs due to the initial burst release but the formulation F5 showed $86.4 \pm 1.22 \%$ drug release in 12 hrs showing sustained release due to increase in polymer concentration, that is, $5 \%$ chitosan. Similarly, for the formulations F6-F10 the drug release was found to be in the range of $85.8 \pm 0.43-96.8 \pm 0.55 \%$ and F10 showing $85.8 \pm 0.43 \%$ drug release as sustained manner in $12 \mathrm{hrs}$ when compared to the other formulations. This is due to increase in the polymer concentration, that is, up to $3.5 \%$ carbopol 974P. Similarly, for the formulations F11-F15, the percentage drug release was found to be in the range of $82.7 \pm 0.23-95.5 \pm 0.83 \%$ and F15 showing $82.7 \pm 0.23 \%$ drug release as the sustained manner in $12 \mathrm{hrs}$ as compared to the other formulations. Hence, this is due to increase in the polymer concentration up to $3.5 \%$ Guar gum. The comparative dissolution studies were conducted for the formulations F5, F10, and F15. Among them, F15 showed $82.7 \pm 0.23 \%$ drug release in 12 hrs in a sustained manner. Hence, the formulation F15 is suitable for sustained release of ropinirole HCL and also showed the better percentage yield, encapsulation efficiency, and swelling properties. The in vitro drug release for the formulations F16 and F17 are 87.5\% and $82.9 \%$, respectively in $12 \mathrm{hrs}$. This showed sustained release due to the combination of chitosan and guar gum in polymer concentrations LL 


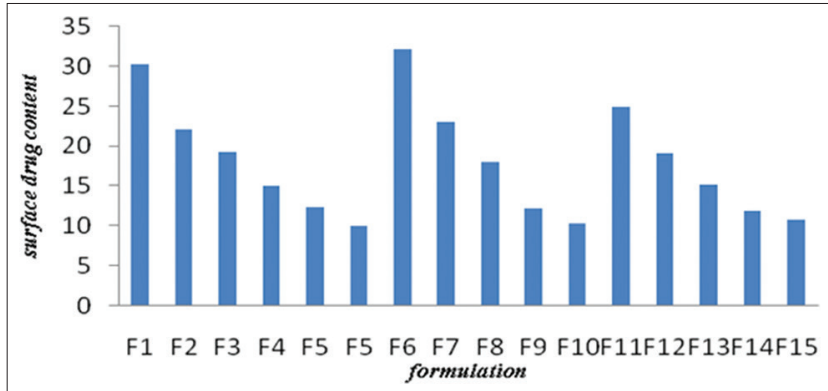

Fig. 4: Comparison of results of surface associated drug content between F1 and F15 formulations

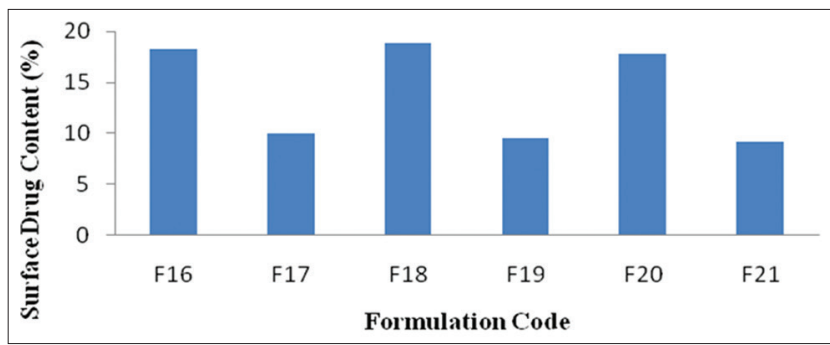

Fig. 5: Comparison of results of surface associated drug content between F16 and F21 formulations

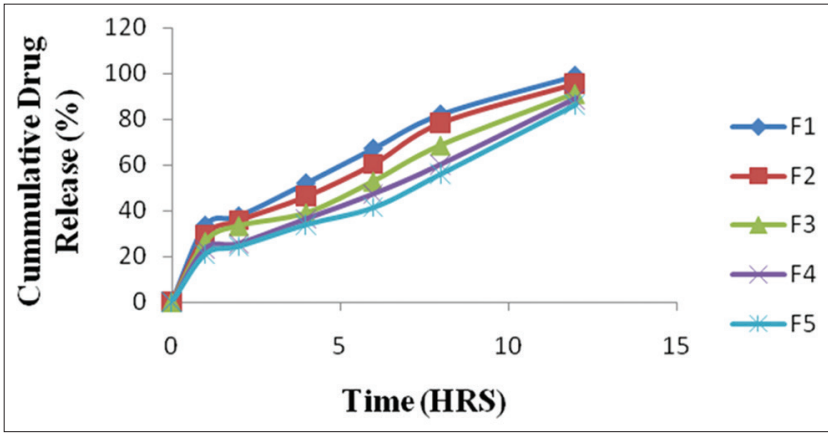

Fig. 6: Graph showing cumulative \% drug release of formulations F1-F5

(3.5\%) and $\mathrm{HH}(5 \%)$, respectively. The drug release for the formulations F18 and F19 in 16 hrs are 89.1\% and 83.6\%, respectively. This showed drug release in a sustained manner due to the combination of polymers chitosan and carbopol 974P in concentrations LL (3.5\%) and HH (5\%), respectively. The drug release for the formulations F20 and F21 in $16 \mathrm{hrs}$ are $85.6 \%$ and $81.2 \%$, respectively. The sustained release in these formulations are due to the combination of polymers carbopol 974P and guar gum in concentrations LL (3.5\%) and $\mathrm{HH}(5 \%)$. The comparative dissolution was run for the formulations F17. F19, F21. Among them, F21 showed $81.2 \%$ drug release in $12 \mathrm{hrs}$ in a sustained manner. Hence, the formulation F21 is also suitable for sustained release of ropinirole HCL using a combination of polymers, guar gum (Table 6-11 and Figs. 6-11).

\section{Drug release kinetics}

The in vitro dissolution data for formulations F1-F21 were analyzed for different kinetic models to find out which drug release mechanism it follows. The values of correlation ( $\mathrm{r}$ ) were calculated and were found to be more linear for first-order release as compared to zero order. The kinetic data were best-fitted to Higuchi model and good regression coefficients were observed.

The kinetic model graphs of F15 formulation and F21 were found best formulation (Table 12 and Figs. 12-21).
Table 5: Data of swelling index of formulations, F1-F21

\begin{tabular}{ll}
\hline Formulation code & Swelling index (in 2 hrs) pH 6.6 \\
\hline F1 & $175.3 \pm 8.07$ \\
F2 & $192 \pm 5.11$ \\
F3 & $215.5 \pm 9.45$ \\
F4 & $240.1 \pm 9.4$ \\
F5 & $286.4 \pm 5.1$ \\
F6 & $169.2 \pm 8.3$ \\
F7 & $200.5 \pm 11.4$ \\
F8 & $255.6 \pm 6.28$ \\
F9 & $282.7 \pm 15.3$ \\
F10 & $311.4 \pm 11.25$ \\
F11 & $159.8 \pm 9.13$ \\
F12 & $185.3 \pm 12.3$ \\
F13 & $248.2 \pm 5.11$ \\
F14 & $290 \pm 7.23$ \\
F15 & $330.5 \pm 11.1$ \\
F16 & $295 \pm 12.1$ \\
F17 & $320 \pm 9.25$ \\
F18 & $270 \pm 8.34$ \\
F19 & $325 \pm 11.2$ \\
F20 & $310 \pm 4.56$ \\
F21 & $350 \pm 9.6$ \\
\hline Fach sample was analyzed in triplicate (n $3 \pm S D), S D:$ Standard deviation
\end{tabular}

Each sample was analyzed in triplicate $(n=3 \pm S D)$, SD: Standard deviation

Table 6: Dissolution profile at $\mathrm{pH} 6.6$ phosphate buffer for ropinirole HCL loaded chitosan microspheres

\begin{tabular}{llllll}
\hline Time (hrs) & F1 & F2 & F3 & F4 & F5 \\
\hline 0 & 0 & 0 & 0 & 0 & 0 \\
1 & 33.5 & 29.2 & 26.5 & 23.4 & 21 \\
2 & 37.6 & 35.7 & 33.4 & 25.7 & 24.4 \\
4 & 52 & 46.2 & 39 & 36.4 & 33.9 \\
6 & 67.1 & 60.4 & 52.9 & 47.3 & 41.4 \\
8 & 82 & 78.3 & 68.6 & 60.2 & 56.1 \\
12 & 98.9 & 95.5 & 91.3 & 89 & 86.4 \\
\hline
\end{tabular}

Table 7: Dissolution profile at $\mathrm{pH} 6.6$ phosphate buffer for ropinirole HCL loaded carbopol 974P microspheres

\begin{tabular}{llllll}
\hline Time (hrs) & F6 & F7 & F8 & F9 & F10 \\
\hline 0 & 0 & 0 & 0 & 0 & 0 \\
1 & 25.5 & 22.5 & 21.2 & 21.3 & 18 \\
2 & 35.8 & 30 & 28.4 & 24.6 & 22.5 \\
4 & 42.7 & 37.8 & 35.1 & 32.5 & 25.6 \\
6 & 74.6 & 63.5 & 61.5 & 46.9 & 35.6 \\
8 & 77.9 & 70.1 & 66.2 & 59.2 & 53.2 \\
12 & 96.8 & 94.3 & 91.1 & 88.1 & 85.8 \\
\hline
\end{tabular}

HCL: Hydrochloride

Table 8: Dissolution profile at pH 6.6 phosphate buffer for ropinirole HCL loaded Guar gum microspheres

\begin{tabular}{llllll}
\hline Time (hrs) & F11 & F12 & F13 & F14 & F15 \\
\hline 0 & 0 & 0 & 0 & 0 & 0 \\
1 & 48 & 36.7 & 24 & 21.07 & 20.4 \\
2 & 54.7 & 40.6 & 34.3 & 29.4 & 27.6 \\
4 & 59.5 & 53.5 & 49.9 & 39.1 & 34 \\
6 & 65.9 & 61.7 & 55.3 & 46.6 & 43.3 \\
8 & 75.8 & 70 & 63.2 & 54.1 & 50.8 \\
12 & 95.5 & 92.8 & 90.1 & 87.5 & 82.7 \\
\hline
\end{tabular}

Scanning electron microscopy (SEM)

Surface morphology and internal cross-sectional structure of the microspheres were investigated with SEM. SEM photomicrographs of 


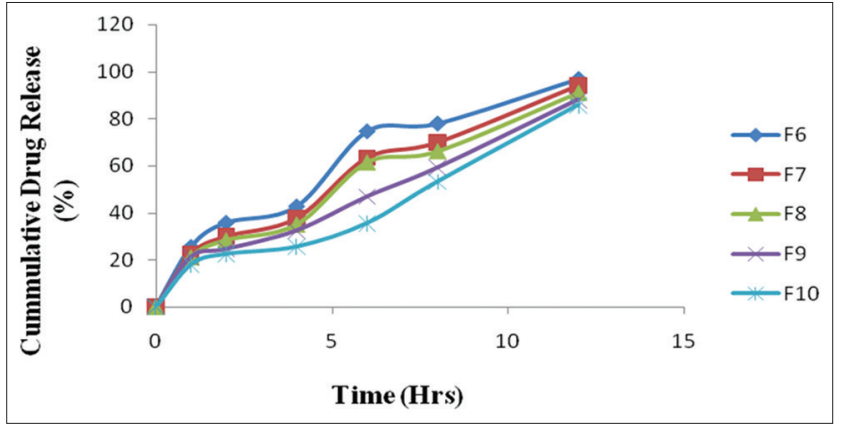

Fig. 7: Graph showing cumulative \% drug release of formulations F6-F10

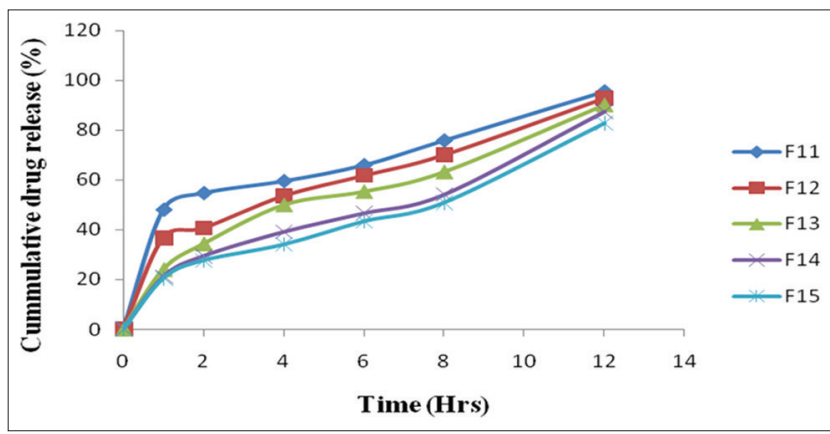

Fig. 8: Graph showing cumulative \% drug release of formulations F11-F15

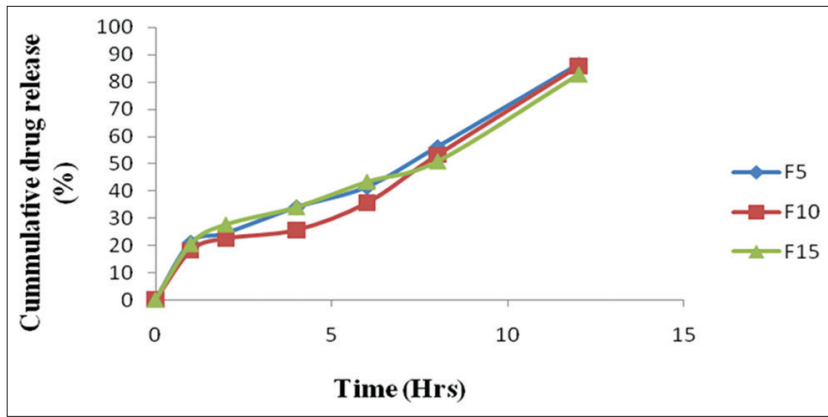

Fig. 9: Comparison of cumulative $\%$ drug release of formulations F5, F10 and F15

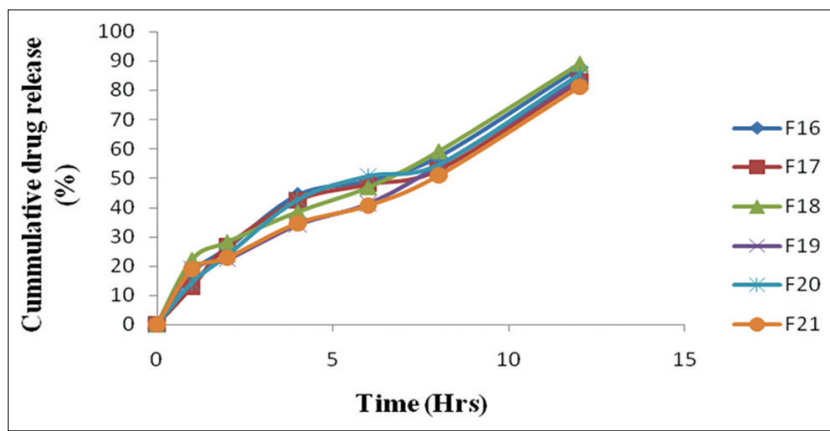

Fig. 10: Graph showing cumulative $\%$ drug release of formulations F16-F21

the optimized formulation are shown in Fig. 22. The microspheres were smooth, spherical, and discrete particles. Very less particulate matter of the drug were seen on the surface of the microspheres indicating uniform distribution of the drug in the polymer network.

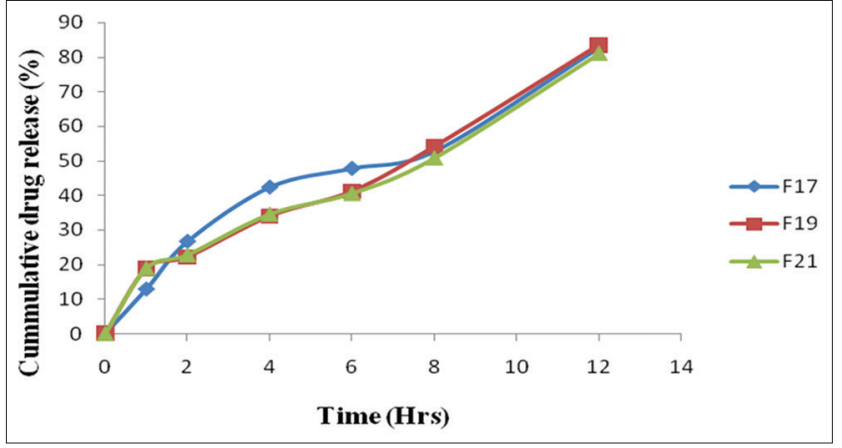

Fig. 11: Comparison of $\%$ cumulative drug release of optimized polymer concentration formulations F17, F19 and F21

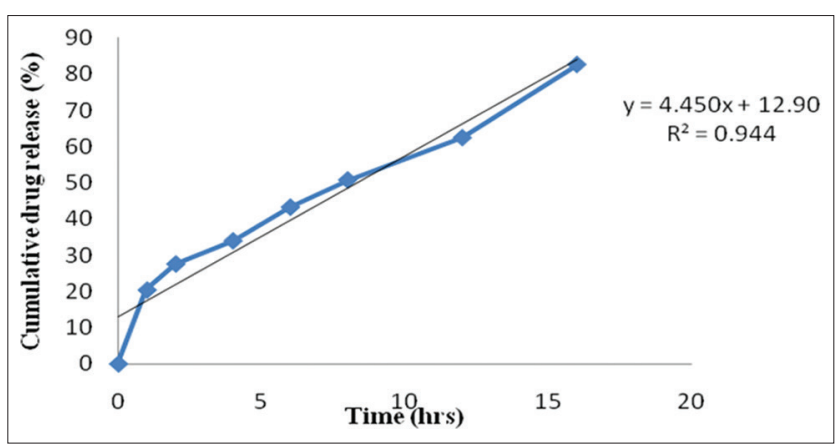

Fig. 12: Zero order release for F15 formulation

Table 9: Dissolution profile at $\mathrm{pH} 6.6$ phosphate buffer for ropinirole HCL loaded optimized formulations

\begin{tabular}{llll}
\hline Time (hrs) & F5 & F10 & F15 \\
\hline 0 & 0 & 0 & 0 \\
1 & 21 & 18 & 20.4 \\
2 & 24.4 & 22.5 & 27.6 \\
4 & 33.9 & 25.6 & 34 \\
6 & 41.4 & 35.6 & 43.3 \\
8 & 56.1 & 53.2 & 50.8 \\
12 & 86.4 & 85.8 & 82.7 \\
\hline HCL: Hydrochloride & & &
\end{tabular}

Table 10: Dissolution profile at $\mathrm{pH} 6.6$ phosphate buffer for ropinirole HCL loaded chitosan - Guar gum, chitosan - carbopol974P and carbopol974P - Guar Gum microspheres

\begin{tabular}{lllllll}
\hline Time (hrs) & F16 & F17 & F18 & F19 & F20 & F21 \\
\hline 0 & 0 & 0 & 0 & 0 & 0 & 0 \\
1 & 17.7 & 12.9 & 22.2 & 18.9 & 14.4 & 18.9 \\
2 & 26.1 & 26.7 & 28.2 & 22.2 & 23.7 & 22.8 \\
4 & 44.2 & 42.4 & 38.5 & 34 & 42.7 & 34.6 \\
6 & 49 & 47.8 & 46.9 & 41.2 & 50.8 & 40.6 \\
8 & 57.2 & 52.8 & 59.2 & 54.2 & 54.7 & 50.9 \\
12 & 87.5 & 82.9 & 89.1 & 83.6 & 85.6 & 81.2 \\
\hline
\end{tabular}

\section{Stability studies}

At the end of stability studies, the microspheres were checked for any changes in physical stability, size, shape, drug content and release profile. Selected formulations like F15 \& F21 microsphere were subjected to exhaustive stability testing at $25 \pm 2{ }^{\circ} \mathrm{C} 60 \pm 5 \% \mathrm{RH}$ for $1^{\text {st }}$ and $2^{\text {nd }}$ month and $40 \pm 2^{\circ} \mathrm{C} 75 \pm 5 \%$ RH for $3^{\text {rd }}$ months. Samples were withdrawn at 1, 2 and 3 months period according to ICH guidelines. 


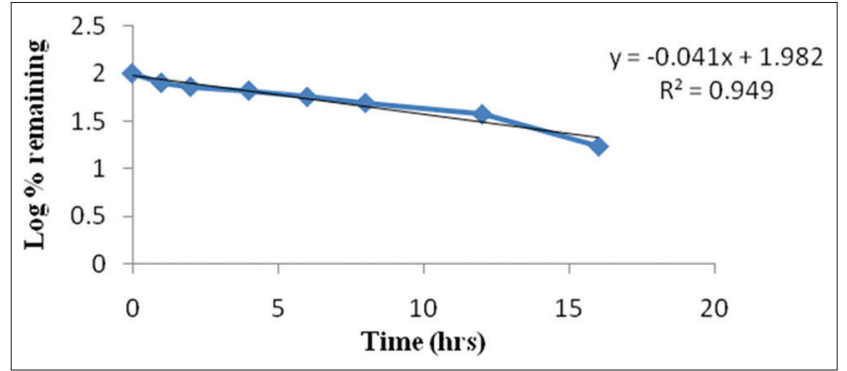

Fig. 13: First order release for F15 formulation

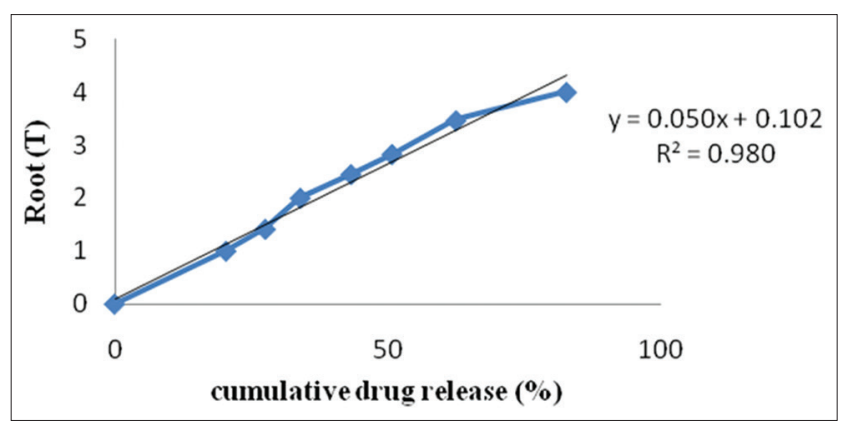

Fig. 14: Higuchi model for F15 formulation

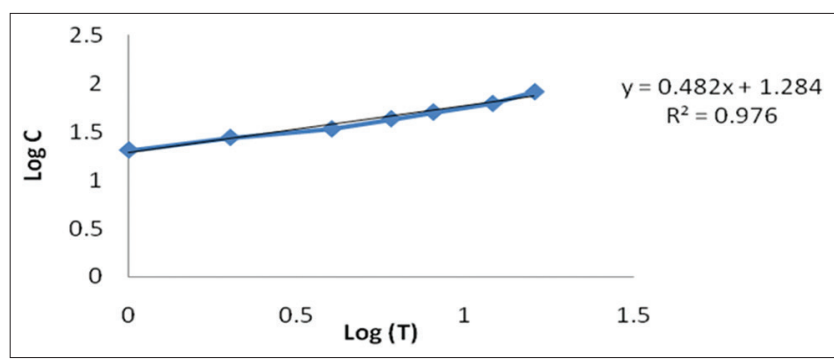

Fig. 15: Korsemeyer peppas model for F15 formulation

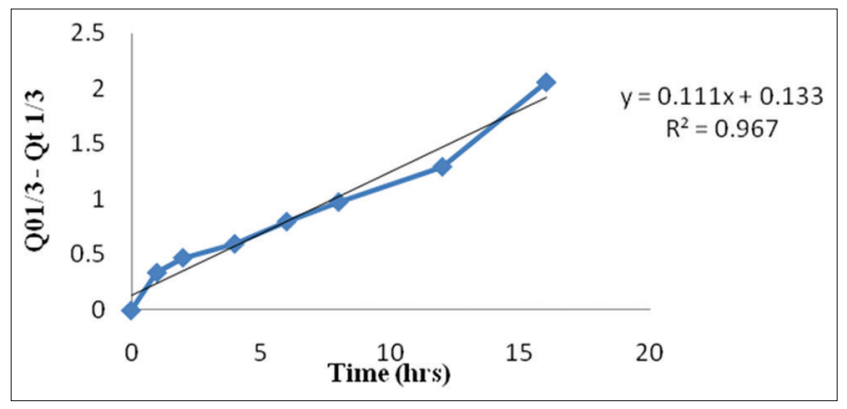

Fig. 16: Hixon crowell model for F15 formulation

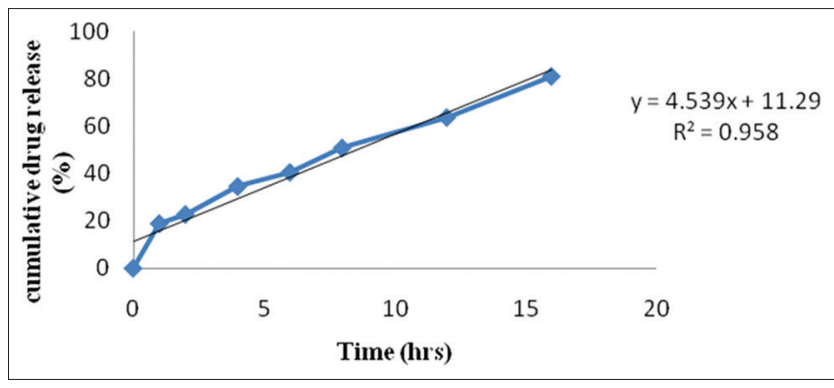

Fig. 17: Zero order release for 21 formulation

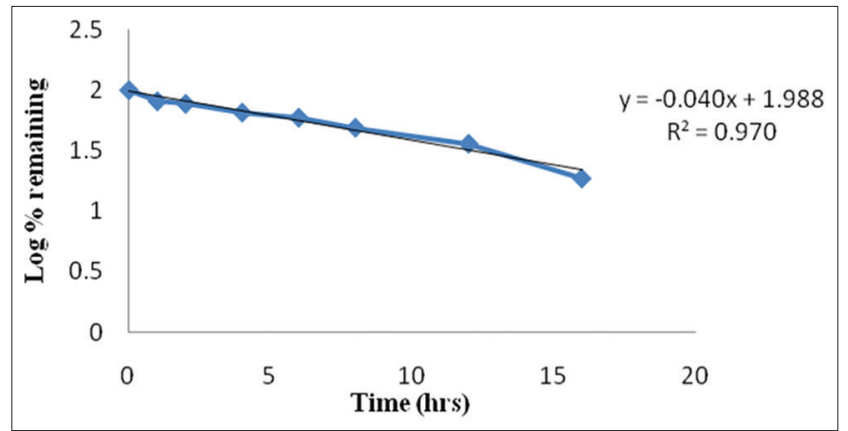

Fig. 18: First order release for 21 formulation

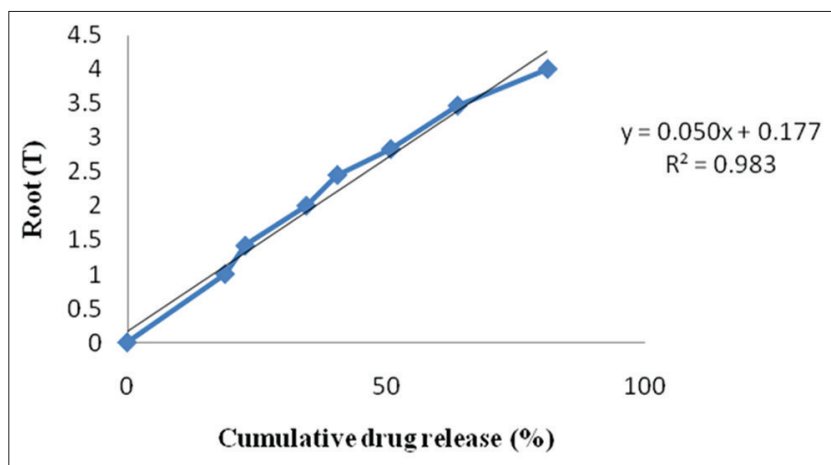

Fig. 19: Higuchi model for F21 formulation

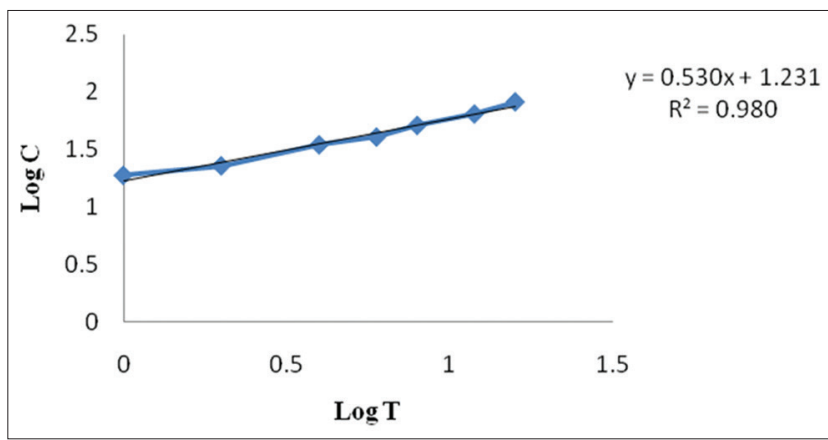

Fig. 20: Korsemeyer Peppas model for F21 formulation

Table 11: Dissolution profile at $\mathrm{pH} 6.6$ phosphate buffer for ropinirole HCL optimized combination formulations

\begin{tabular}{llll}
\hline Time (hrs) & F17 & F19 & F21 \\
\hline 0 & 0 & 0 & 0 \\
1 & 12.9 & 18.9 & 18.9 \\
2 & 26.7 & 22.2 & 22.8 \\
4 & 42.4 & 34 & 34.6 \\
6 & 47.8 & 41.2 & 40.6 \\
8 & 52.8 & 54.2 & 50.9 \\
12 & 82.9 & 83.6 & 81.2 \\
\hline HCL: Hydrochloride & & &
\end{tabular}

The both formulations (F15 and F21) did not show any changes in physical stability, size, shape, drug content and release profile at intermediate conditions. A stability study has been observed in Tables 13 and 14 .

\section{CONCLUSION}

Microspheres were formulated by emulsion solvent evaporation technique using different polymers. Trials were made by changing 
Table 12: In vitro drug release kinetics studies (regression coefficient $\left[\mathrm{r}^{2}\right]$ ) of prepared ropinirole HCL microspheres

\begin{tabular}{|c|c|c|c|c|c|}
\hline Formulation code & Zero $\left(r^{2}\right)$ & First $\left(\mathbf{r}^{2}\right)$ & Higuchi $\left(\mathrm{r}^{2}\right)$ & Korsmeyer Peppas $\left(\mathrm{r}^{2}\right)$ & Hixon Crowell $\left(\mathbf{r}^{2}\right)$ \\
\hline F1 & 0.861 & 0.967 & 0.983 & 0.971 & 0.991 \\
\hline $\mathrm{F} 2$ & 0.883 & 0.988 & 0.984 & 0.974 & 0.982 \\
\hline F3 & 0.915 & 0.983 & 0.986 & 0.963 & 0.985 \\
\hline F4 & 0.95 & 0.976 & 0.985 & 0.962 & 0.99 \\
\hline F5 & 0.96 & 0.957 & 0.976 & 0.962 & 0.979 \\
\hline F6 & 0.855 & 0.98 & 0.966 & 0.955 & 0.973 \\
\hline F7 & 0.909 & 0.973 & 0.981 & 0.969 & 0.985 \\
\hline F9 & 0.957 & 0.974 & 0.98 & 0.096 & 0.989 \\
\hline F10 & 0.972 & 0.947 & 0.944 & 0.922 & 0.972 \\
\hline F11 & 0.739 & 0.962 & 0.922 & 0.947 & 0.935 \\
\hline F12 & 0.839 & 0.977 & 0.979 & 0.978 & 0.967 \\
\hline F13 & 0.894 & 0.983 & 0.996 & 0.995 & 0.98 \\
\hline F14 & 0.94 & 0.942 & 0.987 & 0.988 & 0.968 \\
\hline F15 & 0.944 & 0.949 & 0.98 & 0.976 & 0.967 \\
\hline F16 & 0.935 & 0.978 & 0.993 & 0.992 & 0.986 \\
\hline F19 & 0.964 & 0.981 & 0.981 & 0.976 & 0.991 \\
\hline F20 & 0.93 & 0.972 & 0.988 & 0.984 & 0.978 \\
\hline F21 & 0.958 & 0.97 & 0.983 & 0.98 & 0.982 \\
\hline
\end{tabular}

Each sample was analyzed in triplicate ( $\mathrm{n}=3 \pm \mathrm{SD}$ ), SD: Standard deviation, HCL: Hydrochloride

Table 13: Stability studies of formulation F15 as per ICH Guidelines

\begin{tabular}{|c|c|c|c|c|}
\hline \multirow[t]{2}{*}{ Characteristics } & Initials & 30 days & 60 days & 90 days \\
\hline & $25 \pm 2^{\circ} \mathrm{C} 60 \pm 5 \% \mathrm{RH}$ & $25 \pm 2^{\circ} \mathrm{C} 60 \pm 5 \% \mathrm{RH}$ & $25 \pm 2^{\circ} \mathrm{C} 60 \pm 5 \% \mathrm{RH}$ & $40 \pm 2^{\circ} \mathrm{C} 75 \pm 5 \% \mathrm{RH}$ \\
\hline Drug entrapment efficiency (\%) & $93.7 \pm 1.28$ & $93.4 \pm 0.32$ & $92.9 \pm 1.05$ & $92.5 \pm 0.92$ \\
\hline Swelling index (\%) in pH 6.6 buffer & $330.5 \pm 11.1$ & $325 \pm 8.2$ & $320 \pm 8.0$ & $315 \pm 6.6$ \\
\hline In vitro drug release (\%) & $82.7 \pm 0.23$ & $83.4 \pm 0.32$ & $83.97 \pm 0.71$ & $84.02 \pm 0.36$ \\
\hline
\end{tabular}

HCL: Hydrochloride

Table 14: Stability studies of formulation F21 as per ICH guidelines

\begin{tabular}{|c|c|c|c|c|}
\hline \multirow[t]{2}{*}{ Characteristics } & Initials & 30 days & 60 days & 90 days \\
\hline & $25 \pm 2^{\circ} \mathrm{C} 60 \pm 5 \% \mathrm{RH}$ & $25 \pm 2^{\circ} \mathrm{C} 60 \pm 5 \% \mathrm{RH}$ & $25 \pm 2^{\circ} \mathrm{C} 60 \pm 5 \% \mathrm{RH}$ & $40 \pm 2^{\circ} \mathrm{C} 75 \pm 5 \% \mathrm{RH}$ \\
\hline Drug entrapment efficiency (\%) & $95.3 \pm 2.81$ & $4.3 \pm 0.05$ & $4.3 \pm 0.05$ & $4.3 \pm 0.05$ \\
\hline Swelling index (\%) in pH 6.8 buffer & $350 \pm 9.6$ & $345 \pm 6.3$ & $345 \pm 8.4$ & $342 \pm 4.51$ \\
\hline In vitro drug release $(\%)$ & $81.2 \pm 0.45$ & $81.89 \pm 0.33$ & $82.97 \pm 0.47$ & $83.02 \pm 0.16$ \\
\hline
\end{tabular}

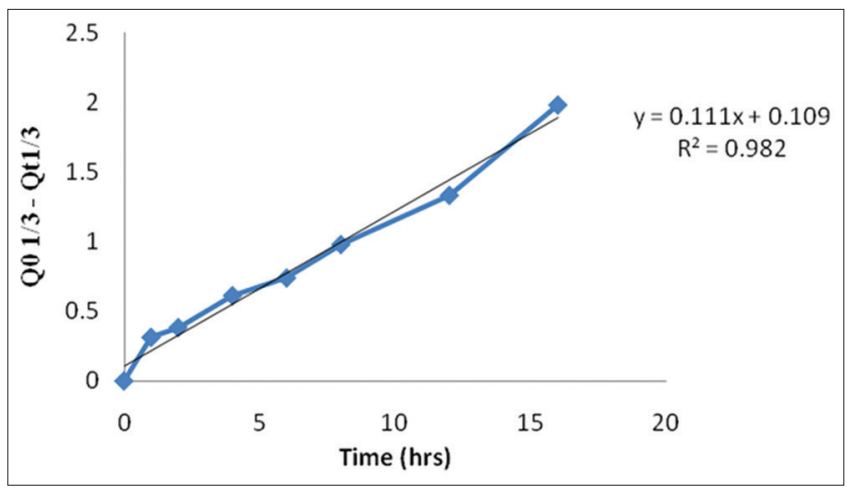

Fig. 21: Hixon crowell model for F21 formulation

the temperature, stirring speeds, concentration of the polymer, Tween-80, Span-80. After so many trials, it was concluded that temperature play a very critical role in the formation of microbeads, it is a continuous process of stirring, with the combination of hydrophilic and lipophilic surfactants. Apart from preventing nasal irritation, the microsphere possesses two major advantages over plain solutions, one is a high solubilization capacity for ropinirole

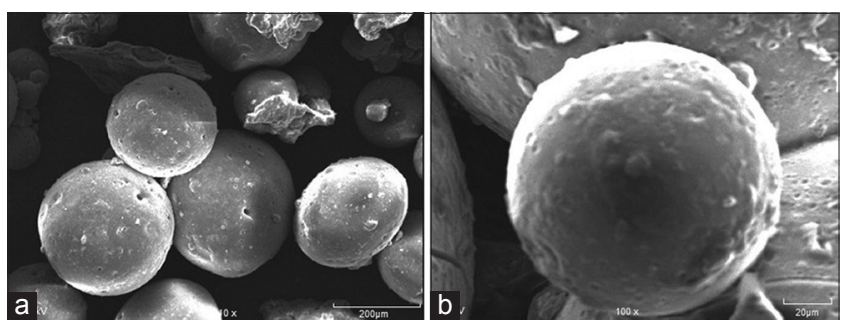

Fig. 22: Scanning electron micrograph of F15 formulation (a) group of microspheres and (b) single microsphere

HCL that exceeds their aqueous solubility and thus allows a reduction in the application volume. The other advantage is that the drug can be rapidly absorbed after nasal administration of the microsphere to meet the emergency treatment purpose. These results indicate that intranasal microsphere of ropinirole may be beneficial for the treatment of Parkinson's disease.

\section{ACKNOWLEDGMENT}

The results described in the paper were part of my Ph.D thesis. Data shown were satisfactory according to Pharmacopoeia. 


\section{REFERENCES}

1. Chaudhari A, Jadhav KR, Kadam VJ. An over view: Microspheres as a nasal drug delivery system. Int J Pharm Sci Rev Res 2010;5(1):8-17.

2. Koyani V, Dedakia A, Matholiya C, Patel P. Microspheres for intranasal delivery system: As review. Bio Inf Drug Targets 2014;2(1):14-9.

3. Sharma A, Khatri K, Patil UK. Nasal route: A potential alternative for antiparkinsonism drug delivery. Int $\mathrm{J}$ PharmTech Res 2008;2(4):2291-306.

4. Pardridge WM. The blood-brain barrier: Bottleneck in brain drug development. NeuroRx 2005;2:3-14

5. Illum L, Watts P, Fisher AN, Hinchcliffe M, Norbury H, Jabbal-Gill I, et al. Intranasal delivery of morphine. J Pharmacol Exp Ther 2002;301:391-400

6. Kisan RJ, Manoj NG, Ishaque MS, Vilarsrao JK, Sambjahi SP. Nasal drug delivery system-factors affecting and applications. Curr Drug Ther 2007;2:27-38

7. Johnson NJ, Leah RH, William HF. Trigeminal pathways deliver a low molecular weight drug from the nose to the brain and orofacial structures. Mol Pharm 2010;7:884.

8. Talegaonkar S, Mishra PR. Intranasal delivery: An approach to bypass the blood brain barrier. Indian J Pharmacol 2004;36:140.

9. Illum L. Nasal drug delivery--possibilities, problems and solutions. J Control Release 2003;21:187.

10. Vyas TK, Babbar AK, Sharma RK, Singh S, Mishra A. Intranasal mucoadhesive microemulsions of clonazepam: Preliminary studies on brain targeting. J Pharm Sci 2006;54:570.

11. Vyas SP, Khar RK. Targeted and Controlled Drug Delivery Novel Carrier Systems. New Delhi: CBS Publishers and Distributers; 2002. p. 489-509.

12. Chang SF, Chein YW. Intranasal drug administration for systemic medication. Pharm Int 1984;5:287.

13. Aspden TJ, Mason JD, Jones NS, Lowe J, Skaugrud O, Illum L. Chitosan as a nasal delivery system: The effect of chitosan solutions on in vitro and in vivo mucociliary transport rates in human turbinates and volunteers. J Pharm Sci 1997;86(4):509-13.

14. Bhosale NR, Hardikar SR, Ashok BV. Formulation and evaluation of transdermal patches of ropinirole $\mathrm{HCl}$. Res J Pharm Bio Chem Sci 2011;2(1):138-48

15. Adnan A, Talegaonkar S, Negi LM, Farhan JA, Khar RK, Zeenat I. Oil based nanocarrier system for transdermal delivery of ropinirole: A mechanistic, pharmacokinetic and biochemical investigation. Int $\mathrm{J}$ Pharm 2011. (Article under Press).

16. Priya S, Koland M, Kumari NS. Formulation and characterization of ropinirole hydrochloride loaded solid lipid nanoparticles. Int J Pharm Pharm Sci 2015;7(9):85-9.

17. Chandrakala V, Saral AM. Quality by design approach in formulation of bioadhesive levodopa microspheres. Int $\mathrm{J}$ Pharm Pharm Sci 2013;5 Suppl 4:172-8 